\title{
A Proposed Opioid Tapering Tool
}

\author{
Benjamin Lai, MB, BCh, BAO, MSc, Daniel Witt, BS, Thomas Thacher, MD, and \\ Terrence Witt, MD
}

Introduction: Previous studies suggest a lack of confidence among primary care providers in managing patients on chronic opioid therapy (COT) for chronic non-cancer related pain (CNCP). The US

Department of Health and Human Services (HHS) recently introduced guidelines on opioid tapering. In light of these recommendations, our group developed an opioid tapering software to assist healthcare providers in managing patients on COT.

Methods: The initial iteration of our software utilizes RedCap for the application programming interface (API). This tool is designed to reduce a patient's prescribed chronic opioids by $5-10 \%$ every month, or 5 morphine milligram equivalents per day (MME), whichever is the greatest, in line with HHS guidelines. Users can also adjust the rate of taper.

Discussion: Our group plans to use this software in an upcoming pilot study to taper patients on COT for CNCP. We are exploring the possibility of transitioning our software into other available APIs with the goal of integrating this software into major electronic health record systems. Our group envisions that our software will provide an additional tool within a patient-centered, multi-modal framework in managing patients on COT for CNCP. ( $\mathrm{J}$ Am Board Fam Med 2020;33:1020-1021.)

Keywords: Electronic Health Records, Opioids, Pain Management, Patient-Centered Care, Pilot Studies, Primary Health Care, Software

Despite increasing attention to the opioid epidemic, many primary care providers lack confidence in prescribing opioids. ${ }^{1-2}$ Evidence regarding the efficacy and safety of chronic opioid therapy (COT) for chronic noncancer pain (CNCP) and the optimal strategy for opioid tapering is limited. ${ }^{3}$ The US Department of Health and Human Services (HHS) recently published guidelines intended to assist providers in tapering opioids. ${ }^{4}$ These guidelines highlight the need for individualizing tapers-noting that some patients may require a slower taper to minimize withdrawal symptoms and increasing success of tapering.

In light of these recommendations, our group has developed an opioid tapering software to assist

This article was externally peer reviewed.

Submitted 19 February 2020; revised 2 May 2020; accepted 18 May 2020.

From the Department of Family Medicine, Mayo Clinic, Rochester, MN (BL, TT); Alix School of Medicine, Mayo Clinic, Rochester, MN (DW); Department of Family Medicine, Mayo Clinic Health Systems-Northwest Wisconsin, EauClaire, WI (TW).

Funding: None.

Conflict of interest: None.

Corresponding author: Benjamin Lai, MB, BCh, BAO, MSc, Department of Family Medicine, 200 1st St SW, Rochester, MN 55905 (E-mail: lai.benjamin@mayo.edu). clinicians in managing patients on COT deemed appropriate for tapering. The software is designed to reduce a patient's prescribed chronic opioids by $5 \%$ to $10 \%$ every month (rounded based on available tablet strength), or 5 morphine milligram equivalents per day (MMED), whichever is the greatest, in line with HHS guidelines. The program, however, allows users to adjust the rate of taper; it also accounts for patients taking multiple opioids, including combinations of long- and short-acting opioids (including those combined with acetaminophen). The software will generate a tapering schedule based on a patient's initial daily opioid dose, frequency of dosing, and available medication dosage formulation. The software utilizes the following rules to generate a taper:

- When multiple opioids are used, 1 medication will be tapered to $0 \mathrm{mg}$ before initiating the taper of the next medication.

- The tool will identify and initiate taper of extended-release medications before immediaterelease medications.

- If there are multiple extended-release or immediate-release medications for a given patient, the tool will use the following tapering hierarchy based on estimated risk of harm (higher risk of 
harm tapered earlier): oxycodone $>$ hydromorphone $>$ morphine $>$ hydrocodone $>$ codeine $>$ tramadol. We chose to taper tramadol last due to its weaker opioid effect and possibly lower abuse potential compared with other opioids. ${ }^{5}$

- If there are multiple daily doses for a single medication, all doses will be concurrently lowered rather than tapering 1 dose at a time-as long as the taper adheres to our tapering rules (ie, $5 \%$ to $10 \%$ per month, or $5 \mathrm{MMED}$, whichever is greater). If concurrent tapering of multiple doses of a single medication cannot be achieved due to tapering rules, then episodes earlier in the day will be discontinued before episodes later in the day.

We are currently using RedCap for the application programming interface in the initial iteration of this software (https://www.project-redcap.org/). RedCap is a Web-based application used to develop and manage surveys and databases; information collected and stored in this environment is secure and HIPAA compliant. Our group intends to use this software in an upcoming pilot study to taper patients on COT for CNCP.

We realize that the initial iteration of our software does have some limitations. For example, this tool does not provide tapering schedules for patients on some opioid formulations, including fentanyl and methadone. Our tool also does not account for concurrent chronic benzodiazepine use when generating tapering schedules. Moreover, our tapering hierarchy has limited support in the literature.

We are exploring the possibility of transitioning our software into other available application programming interfaces (eg, App Orchard in Epic) with the goal of integrating this software into major electronic health record systems. We invite readers with experience using RedCap in research or clinical settings to provide recommendations on incorporating these additional features into our tool, making it more robust and applicable in diverse clinical scenarios.

Our group envisions that our software will provide clinicians an additional tool within a patient-centered, multimodal framework in managing patients on COT for CNCP.

To see this article online, please go to: bttp://jabfm.org/content/ 33/6/1020.full.

\section{References}

1. Jamison RN, Sheehan KAS, Matthews M, Ross EL. Beliefs and attitudes about opioid prescribing and chronic pain management: survey of primary care providers. J Opioid Manag 2014;10:375-82.

2. Toye F, Seers K, Tierney S, Barker KL. A qualitative evidence synthesis to explore healthcare professionals' experience of prescribing opioids to adults with chronic non-malignant pain. BMC Fam Pract 2017;18:94.

3. Chou R, Turner JA, Devine EB, Hansen RN, Sullivan SD, Blazina I, et al. The effectiveness and risks of long-term opioid therapy for chronic pain: a systematic review for a National Institutes of Health pathways to prevention workshop. Ann Intern Med 2015;162:276-86.

4. HHS Guide for Clinicians on the Appropriate Dosage Reduction or Discontinuation of LongTerm Opioid Analgesics. September 2019. Available from: https://www.drugabuse.gov/nidamed-medicalhealth-professionals/opioid-crisis-pain-management/ hhs-guide-clinicians-appropriate-dosage-reductionor-discontinuation-long-term-opioid.

5. Adams EH, Breiner S, Cicero TJ, Geller A, Inciardi JA, Schnoll SH, et al. A comparison of the abuse liability of tramadol, NSAIDs, and hydrocodone in patients with chronic pain. J Pain Symptom Manage 2006;31:465-76. 